\title{
Traspaso del tipo de cambio al nivel de precios: Un análisis regional
}

\section{Exchange rate pass-through on prices: A regional analysis}

\author{
Ramón A. Castillo-Ponce* \\ Rogelio Varela-Llamas** \\ Juan Manuel Ocegueda-Hernández**
}

\begin{abstract}
We evaluate the pass-through of the exchange rate on the price level in Mexico. We consider the national consumer price index and regional price indexes. The analysis is implemented for the long-and short-run. The results show that the pass-through has decreased at national and regional levels for both time horizons. In the long-run the reduction is similar across regions; in the short-run, however, this is different. While in some regions the transitory effect persists, in others it does not. This suggests that each region exhibits specific socioeconomic characteristics that produce distinct inflationary dynamics.
\end{abstract}

Keywords: exchange rate pass-through, regional inflation, co-integration, common cycles.

\section{Resumen}

En esta investigación se evalúa el traspaso del tipo de cambio al nivel de precios en México, asimismo, se considera el índice nacional de precios al consumidor e índices regionales. El análisis se instrumenta para el largo y corto plazos, los resultados indican que el traspaso ha disminuido tanto a nivel nacional como regional en ambos horizontes. En el largo plazo la disminución es similar a través de regiones; en el corto, la misma es marcadamente distinta, mientras que en algunas regiones el efecto transitorio persiste, en otras desaparece. Esto sugiere que cada región exhibe características socioeconómicas específicas que conllevan a dinámicas inflacionarias distintas entre sí.

Palabras clave: Traspaso de tipo de cambio, inflación regional, cointegración, ciclos comunes.

* Universidad Autónoma de Baja California, México y Universidad Estatal de California, Los Ángeles. Correo-e: rcastil@calstatela.edu

** Universidad Autónoma de Baja California, México. Correos-e: rvarela@uabc.edu.mx, jmocegueda@uabc.edu.mx 


\section{Introducción}

La inflación es un fenómeno que por décadas ha capturado la atención de académicos y de funcionarios o diseñadores de políticas públicas, cuyo cargo les exige tomar decisiones que repercuten en el desarrollo social y económico de la población. La razón es clara, alzas en los precios influyen negativamente sobre el bienestar de la sociedad. En la literatura económica se encuentra una amplia gama de estudios sobre el tema, particularmente para países desarrollados. Documentos de investigadores como Solow (1969) y Lucas y Rapping (1969) marcaron la pauta en esta línea de investigación; con respecto a países en vías de desarrollo, el interés sobre la dinámica del nivel de precios tomó importancia a partir de mediados de la década de los setenta, en gran medida como respuesta a los periodos hiperinflacionarios que varias economías sufrieron entonces. ${ }^{1}$

Para el caso específico de México, el estudio de este fenómeno ha sido relativamente escaso, dentro de las temáticas que se han abordado se pueden destacar tres: el análisis de los determinantes de la inflación, el efecto de las variaciones del tipo de cambio sobre el nivel de precios, y la relación que existe entre la inflación y el desempeño económico. Sobre el primer enfoque se puede mencionar a Fullerton y Calderón (1999), quienes estiman un modelo monetario de inflación. Garcés (1999) y Esquivel y Razo (2003), por otra parte, muestran que los salarios y el tipo de cambio influyen significativamente sobre el nivel de precios; con respecto al segundo acercamiento se encuentran los trabajos de Conesa (1998) y Castillo y Herrera (2008), que determinan la importancia del traspaso del tipo de cambio a los precios. En ambos casos se concluye que dicho efecto depende de factores como la estructura de mercado y la apertura externa de las industrias: entre mayor sea la concentración industrial o la exposición al exterior, mayor es el efecto del tipo de cambio. Finalmente, Pérez y Schwartz (1999) evalúan el impacto de la inflación sobre distintas variables macroeconómicas; consistente con la teoría, los autores muestran que la inflación afecta negativamente a la actividad económica.

A partir de la revisión de la literatura se aprecia que ésta se ha enfocado en el análisis a nivel agregado, poniendo escaso interés en la dinámica inflacionaria a nivel regional. ${ }^{2}$ Así, en este documento se plantea contribuir al debate con un estudio de la inflación para diferentes zonas geográficas de México. ¿Por qué puede ser esto interesante? Fundamentalmente porque se conoce muy poco sobre el tema como se indicó en el párrafo an-

\footnotetext{
(1967)

' Para el periodo anterior a los setenta se destacan estudios como el de Harberger (1963) y Baer

${ }^{2}$ Holmes (2008) y Kamin y Klau (2003) realizan estudios sobre el comportamiento de la inflación a nivel regional, pero no consideran el caso específico de México.
} 
terior, documentos de autores como Garcés (1999) y Esquivel y Razo (2003) han establecido que existe un efecto significativo de las variaciones en el tipo de cambio y los salarios sobre el índice nacional de precios al consumidor, pero no se conoce si dicho efecto es homogéneo en toda la república. Una excepción que se aproxima al análisis que aquí se plantea es el documento de Sonora (2005), que evalúa el comportamiento de los precios a nivel de ciudades de la república mexicana. A diferencia del presente estudio, en él se analiza la paridad del poder de compra.

De las variables que se han identificado como determinantes de la inflación en México, el tipo de cambio destaca como un candidato ideal para evaluar si el comportamiento de los precios es distinto en diferentes zonas del país, en particular se considera la dinámica de la frontera norte en cuanto a la circulación del dólar y la demanda transfronteriza de bienes y servicios. Razonablemente, se puede anticipar que una depreciación del peso, por ejemplo, tendría un efecto distinto sobre el nivel de precios en esta región con respecto a otras zonas geográficas del país; ciertamente, la depreciación llevaría a un cambio en la demanda de bienes y servicios, en ciudades como Tijuana y Ciudad Juárez, diferente al que se observaría en Iguala o Toluca, de igual forma, los cambios en los costos de producción de las empresas, en respuesta a variaciones en el tipo de cambio, podrían ser distintos en el norte y en el sur. En consecuencia, el comportamiento de los precios regionales no tendría porqué ser idéntico, el mismo estaría condicionado a las características socioeconómicas individuales de cada región. ¿Cómo sería?, es la pregunta que se plantea contestar en este documento.

Para acotar el estudio, el análisis se enfoca en identificar el efecto de las variaciones del tipo de cambio nominal sobre los precios para dos periodos: antes y después de 1995, año en el que se modificó el sistema de tipo de cambio en el país. En este sentido, el estudio es similar en espíritu al de Baqueiro et al. (2003), que determina el nivel de traspaso del tipo de cambio al nivel de precios, bajo diversos escenarios de inflación. En dicho documento, los autores encuentran que el traspaso disminuye a medida que la inflación desciende. Así, el presente análisis busca determinar si, a partir de la adopción del régimen de libre flotación, el traspaso del tipo de cambio a los precios se ha modificado; ello, en una evaluación a nivel regional.

Evidentemente el tipo de cambio no es el único factor que influye sobre los precios y, en consecuencia, una especificación que sólo incluya dicha variable no se puede entender como completa. Para los propósitos de la presente investigación, sin embargo, no es necesario especificar una ecuación multivariada de la inflación ya que, únicamente, se desea determinar si la magnitud del traspaso del tipo de cambio se ha modificado a 
partir de 1995. Así, a pesar de que pudiera ser que los coeficientes obtenidos se encuentren sobrestimados, las conclusiones se centrarán en las magnitudes relativas antes y después del cambio de régimen, y no sobre la magnitud en sí misma. ${ }^{3}$

Para el ejercicio econométrico que se presenta se consideran estimaciones de largo y corto plazo entre las variables de interés. De esta forma no sólo se identifican similitudes en las tendencias de las series, sino su respuesta conjunta a choques transitorios. En este sentido, el ejercicio que se lleva a cabo es marcadamente distinto a los que aparecen en la literatura, los cuales se enfocan en el análisis tendencial; mientras que es interesante entender el traspaso del tipo de cambio a los precios en el estado estacionario, resulta igualmente importante conocer la respuesta instantánea de la inflación a cambios no permanentes en los precios externos. Las metodologías que se proponen para llevar a cabo el estudio son estándar en el quehacer econométrico. Para el análisis de largo plazo se emplea la sugerida por Johansen (1991), y para la estimación de movimientos transitorios comunes se implementa la desarrollada por Vahid y Engle (1993).

En la sección metodológica se mencionan algunos particulares sobre ambas; el resto del documento se estructura de la siguiente manera: en la primera sección se describen los datos y se presentan ilustraciones de su comportamiento, en la segunda sección se lleva a cabo el ejercicio econométrico y se discuten los resultados, finalmente se exponen las conclusiones a las que se llegaron con este análisis.

\section{Datos y análisis gráfico}

Los datos que se emplean se refieren a índices de precios y el tipo de cambio nominal, para los primeros se obtuvo información a nivel nacional e índice nacional de precios al consumidor para siete regiones de la república mexicana: frontera norte, zona noroeste, zona noreste, centro norte del país, centro sur, área metropolitana de la ciudad de México y zona sur. El tipo de cambio concierne al denominado fecha de liquidación, que corresponde cercanamente al llamado spot exchange que es el que tradicionalmente se emplea en estimaciones empíricas. ${ }^{4} \mathrm{La}$ fuente de las variables fue Banco de México, el periodo de estudio abarca enero de 1982 a diciembre de 2007, la frecuencia de los datos es mensual. En el cuadro 1 se muestra la descripción de cada región en cuanto a las ciudades que son consideradas para el cálculo del índice de precios.

\footnotetext{
${ }^{3}$ Cabe resaltar que, al respecto, el presente análisis es similar al realizado en Baqueiro $e t$ al. (2003). Al igual que aquí, los autores únicamente incluyeron al tipo de cambio como variable explicativa del nivel de precios.

${ }^{4}$ Véase Dornbusch (1976) para el argumento teórico.
} 


\section{Cuadro 1 \\ Regiones de análisis}

\begin{tabular}{|c|c|c|c|}
\hline Región & Ciudad & Región & Ciudad \\
\hline \multirow[t]{7}{*}{ Frontera norte } & Ciudad Acuña & Centro norte & Aguascalientes \\
\hline & Ciudad Juárez & & Colima \\
\hline & $\mathrm{La} \mathrm{Paz}$ & & Cortazar \\
\hline & Matamoros & & Guadalajara \\
\hline & Mexicali & & Jacona \\
\hline & Tijuana & & León \\
\hline & & & Morelia \\
\hline \multirow[t]{5}{*}{ Noroeste } & Culiacán & & Querétaro \\
\hline & Hermosillo & & San Luis Potosí \\
\hline & Huatabampo & & Tepatitlán \\
\hline & Tepic & & \\
\hline & & Centro sur & Acapulco \\
\hline \multirow[t]{16}{*}{ Noreste } & Ciudad Jiménez & & Córdoba \\
\hline & Chihuahua & & Cuernavaca \\
\hline & Durango & & Veracruz \\
\hline & Fresnillo & & Iguala \\
\hline & Monclova & & Puebla \\
\hline & Monterrey & & San Andrés Tuxtla \\
\hline & Tampico & & Tlaxcala \\
\hline & Torreón & & Toluca \\
\hline & & & Tulancingo \\
\hline & & Sur & Campeche \\
\hline & & & Chetumal \\
\hline & & & Mérida \\
\hline & & & Oaxaca \\
\hline & & & Tapachula \\
\hline & & & Villahermosa \\
\hline & & & Tehuantepec \\
\hline
\end{tabular}

Fuente: Elaboración propia con datos del Banco de México (2008).

El ejercicio inicia con una breve descripción del comportamiento de las series, las cuales se presentan en las gráficas i y ir. En la primera de ellas se observan las variables en niveles. En todos los casos la escala de la izquierda mide al tipo de cambio nominal y la de la derecha al índice de precios. En general, la dinámica de los precios es similar a través de las distintas regiones, se aprecia claramente la devaluación de finales de 1994 y el correspondiente cambio en la pendiente de las series de precios que indica un alza en la inflación. Es interesante que a partir del 2003 se observen ciertas diferencias en el comportamiento de los precios. Por 
ejemplo, en la zona noroeste la serie presenta una marcada estacionalidad. Asimismo, para el caso de la frontera norte se aprecia una serie con fluctuaciones más marcadas con relación al resto de las regiones.

En la gráfica II se muestran las tasas de crecimiento o variaciones anuales. Aquí es clara la relación que existe entre las depreciaciones/apreciaciones del tipo de cambio y la inflación. En la totalidad de las gráficas los movimientos de las dos variables se encuentran positivamente relacionados, por ejemplo, la fuerte depreciación de principios de los ochenta se ve acompañada de una significativa alza en la inflación, que alcanzó tasas por arriba de 100\%, de igual forma, la devaluación de 1995 se asocia con un incremento en los precios que habían experimentado una tendencia a la baja desde 1989. Finalmente, con la adopción del sistema de tipo cambiario flexible, se nota una caída sostenida de la inflación.

La relación antes descrita entre el tipo de cambio y los precios es claramente consistente con lo que indica la teoría económica, de acuerdo con ella, una depreciación, por ejemplo, aumenta los costos de los insumos importados y por ende los costos totales de producción. Este incremento en los costos es en turno traspasado a los precios que paga el consumidor y se refleja, naturalmente, en un incremento en la inflación. Para el caso particular de México, el argumento es aún más convincente, ya que debido a los altos índices de concentración de mercado que se encuentran en el país el traspaso de los precios-productor a los precios-consumidor suele ser elevado, lo cual no se observa en economías que se caracterizan por tener estructuras de mercado más competitivas. ${ }^{5}$

Cabe señalar que a simple vista no se observa una diferencia sustancial en el comportamiento de los precios o las tasas de inflación entre las regiones, lo cual, por supuesto, no implica que sus dinámicas estocásticas también se comporten de manera similar, para determinar esto con precisión en la siguiente sección se lleva a cabo el análisis formal.

\section{Ejercicio econométrico}

\subsection{Metodología}

Como se indicó, en esta sección se propone implementar dos metodologías, la primera se refiere a la prueba de cointegración sugerida por Johansen (1991), y la segunda a la prueba de ciclos comunes que se

${ }^{5}$ Conesa (1998) describe puntualmente la relación que existe entre la estructura de mercado y el nivel de traspaso de tipo de cambio. Brevemente: incrementos en los costos de producción resultan en alzas en los precios al consumidor más significativas cuando la estructura de mercado es altamente concentrada. Esto es así porque a un monopolio, por ejemplo, le es relativamente más fácil traspasar el incremento de los costos al precio de su producto, en relación con lo que podría hacer una empresa que opera en un mercado altamente competitivo. 


\section{Gráfica I}

\section{Tipo de cambio y precios en niveles: nacional y por región}
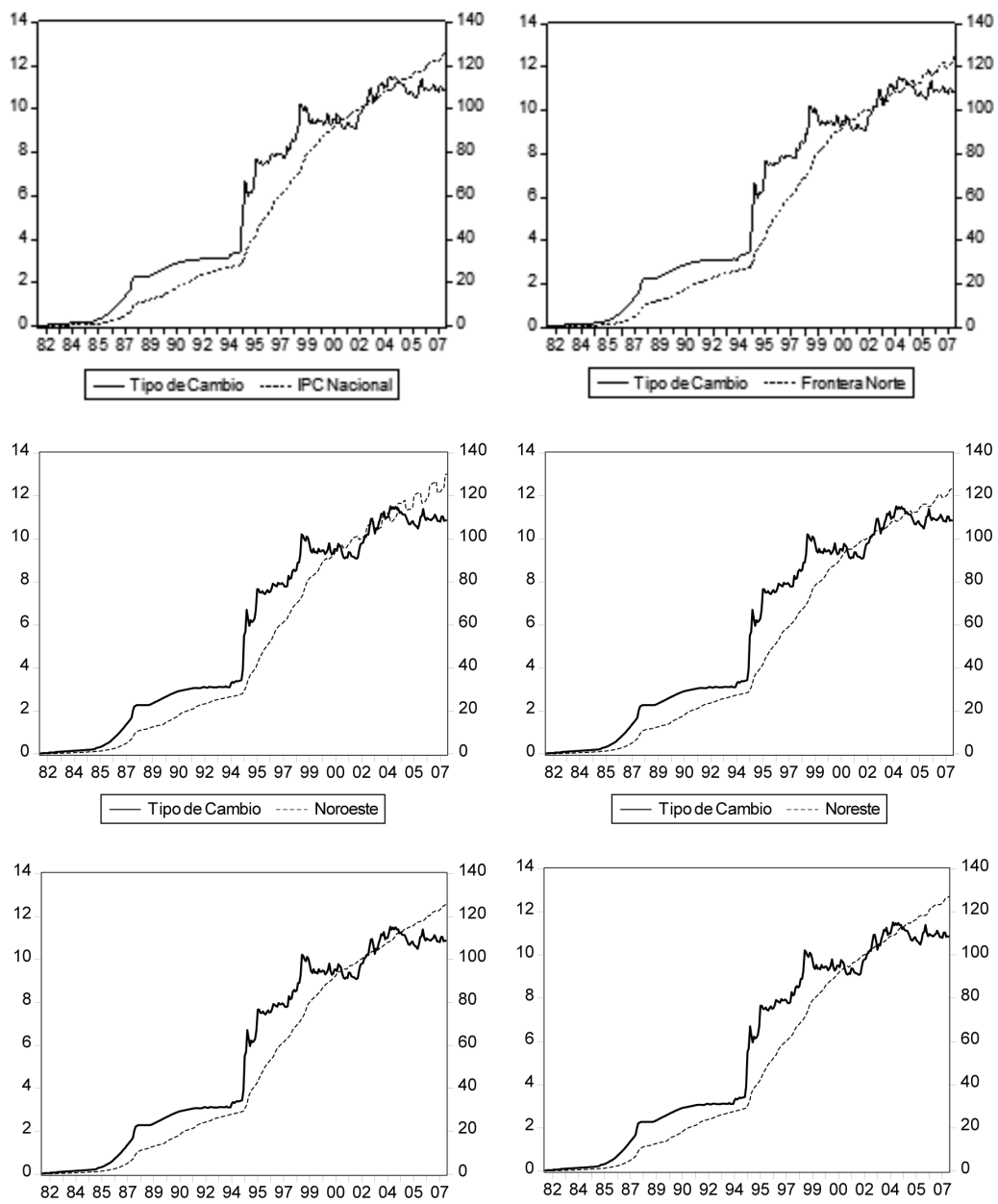

- Tipo de Cambio ---- Centro Norte

- Tipo de Cambio --.-- Centro Sur
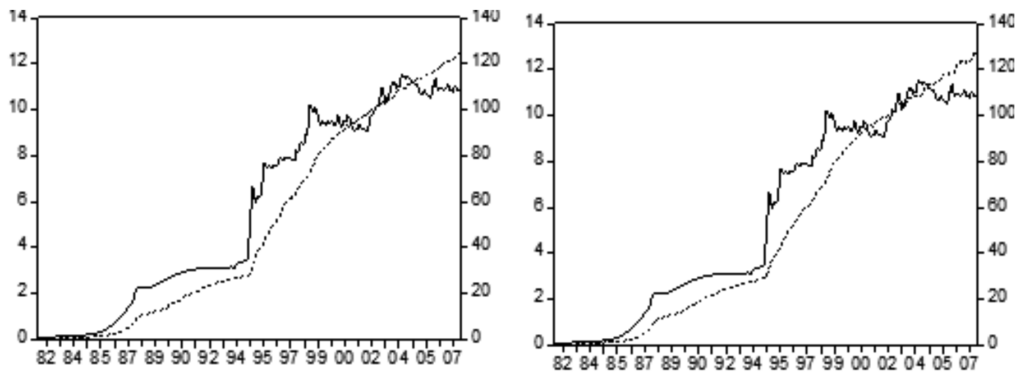

- Tipo de Cambio -.... Metropoltana

- T ipode Cambio $\cdots \cdot-\cdot$ Sur

Fuente: Elaboración propia con datos del Banco de México (2008). 


\section{Gráfica II}

Tipo de cambio y precios en variación anual: nacional y por región
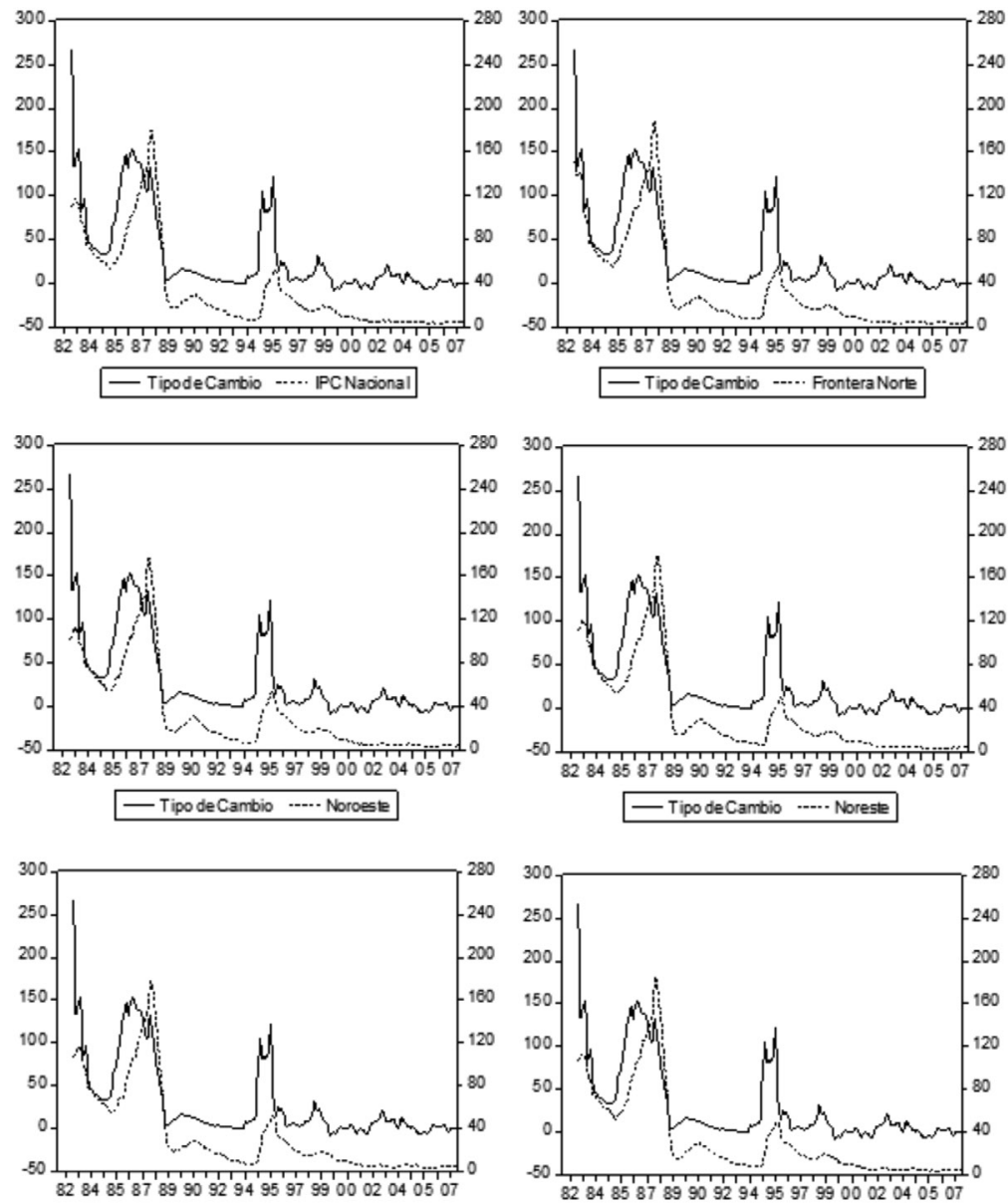

- TipodeCambio -..... CentroNorte
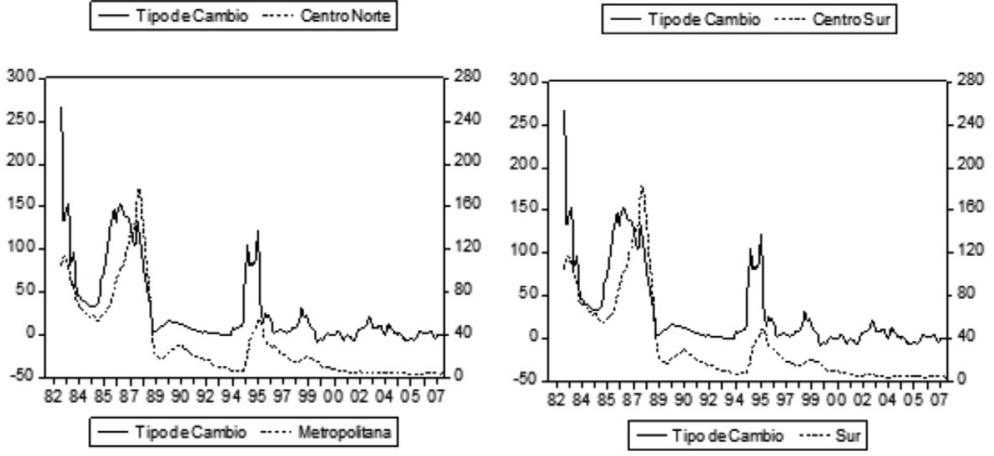

Fuente: Elaboración propia con datos del Banco de México (2008). 
encuentra en Vahid y Engle (1993). Debido a que la metodología de cointegración es ampliamente conocida no se incluye una descripción de la misma; La pertinente a los ciclos comunes se ilustra, brevemente, a continuación: Considere la representación Wold de un vector $y_{t}$ de dimensión $n x 1$ que es estacionario en primeras diferencias:

$$
\Delta y_{t}=C(L) e_{t}=C(1) e_{i}+(1-L) C^{*}(L) e_{t}
$$

Si se integra la ecuación (1) se obtiene:

$$
y_{t}=C(1) \sum_{\mathrm{i}=0}^{\infty} e_{t-i}+C^{*}(L) e_{t}
$$

Esta expresión es similar a la que presentan Stock y Watson (1988) para representar la descomposición de una serie de tiempo. El primer término de la derecha en la expresión (2) representa la parte tendencial, mientras que el segundo término se refiere a la parte cíclica estacionaria. En caso de existir cointegración se tendría que $\alpha^{\prime} C(1)=0$ y la matriz $\alpha$ contendría los $r$ vectores de cointegración. De igual forma, si las series comparten ciclos comunes, sería el caso que $\tilde{a}^{\prime} C^{*}(L)=0$ y la matriz ã incluiría los $s$ vectores de comovimiento o ciclo común. La metodología de Johansen permite determinar $r$ explícitamente. La prueba de ciclos comunes propuesta por Vahid y Engle (1993) identifica $s$. Los autores plantean estimar las correlaciones canónicas al cuadrado del sistema, $\lambda^{2}$, y después realizar una prueba de hipótesis en la cual la hipótesis nula es $\lambda^{2}{ }_{j}=0, \forall=1,2, \ldots, s$. En este caso el estadístico de prueba es $C(p, s)=-(T-$ $p-1) \sum_{j=1}^{s} \log \left(1-\lambda^{2}\right)$ y se encuentra distribuido $\chi^{2} \operatorname{con} s^{2}+s n p+s r-s n$ grados de libertad. El término $n$ se refiere al número de variables en el sistema y $p$ al número de rezagos óptimos en primeras diferencias.

El ejercicio econométrico que se presenta a continuación considera la mecánica descrita anteriormente, de tal manera que se llevan a cabo pruebas de raíz unitaria, de cointegración y de ciclo común en este orden.

\subsection{Naturaleza estocástica de las series}

Como primer ejercicio se instrumentan pruebas de raíz unitaria. Se emplea la metodología sugerida por Kwiatkowski-Phillips-Schmidt-Shin (KPSs); ya que algunos autores señalan que puede tener cierta ventaja sobre otras pruebas en su poder de detección. ${ }^{6}$ Los resultados se presentan en el cuadro 2. Primero, se destaca que para el periodo anterior a la modificación de régimen cambiario, de 1982 a 1994, la totalidad de las series se

\footnotetext{
${ }^{6}$ Véase Patterson (2000).
} 
encuentra integrada de orden 1, I(1). Para el periodo posterior se obtiene evidencia mixta. Las únicas dos variables que resultan ser I(1) son el tipo de cambio y los precios de la región noroeste, el resto parecen ser series integradas de orden 2; dado esto, se opta por implementar pruebas alternativas para evaluar más profundamente el comportamiento estocástico de las series, después de todo, es razonable suponer que la inflación es un proceso estacionario y por ende el nivel de precios un proceso no estacionario. De hecho, si se observan las ilustraciones de la gráfica II, es posible notar que el comportamiento de la inflación a partir de 1996 es similar al de una serie con media constante. Así, en el cuadro 2 se reportan los resultados de la prueba Dickey Fuller Aumentada (DFA). De acuerdo con ellos, las series de precios están efectivamente integradas de orden 1.

\section{Cuadro 2}

Pruebas de raíz unitaria

\begin{tabular}{lcccccc}
\hline & \multicolumn{3}{c}{ Prueba KPSS } & \multicolumn{2}{c}{ Prueba DFA } \\
\hline \multirow{2}{*}{ Serie } & \multicolumn{2}{c}{ 1982-1994 } & \multicolumn{2}{c}{ 1996-2007 } & \multicolumn{2}{c}{ 1996-2007 } \\
\cline { 2 - 7 } & niveles & diferencia & niveles & diferencia & niveles & diferencia \\
\hline \multirow{2}{*}{ Tipo de cambio critico 0.15} & \multicolumn{2}{c}{ Valor critico 0.15} & \multicolumn{2}{c}{ Valor critico -3.44} \\
IPC nacional & 0.37 & 0.14 & 0.16 & 0.05 & & \\
Frontera norte & 0.36 & 0.12 & 0.36 & 0.31 & -2.87 & -4.42 \\
Noroeste & 0.36 & 0.10 & 0.36 & 0.26 & -2.76 & -5.12 \\
Noreste & 0.36 & 0.12 & 0.36 & 0.14 & & \\
Centro norte & 0.36 & 0.11 & 0.35 & 0.26 & -2.65 & -4.55 \\
Centro sur & 0.36 & 0.12 & 0.36 & 0.34 & -2.72 & -4.00 \\
Metropolitana & 0.36 & 0.12 & 0.34 & 0.32 & -2.53 & -4.05 \\
Sur & 0.36 & 0.13 & 0.36 & 0.35 & -2.71 & -4.02 \\
\hline
\end{tabular}

Fuente: Elaboración propia.

\subsection{Análisis de largo plazo}

Una vez determinado el orden de integración se procede a realizar las pruebas de cointegración. Se construyen sistemas bivariados y se incluye al tipo de cambio con el respectivo índice de precios para cada una de las ocho series consideradas. Los resultados para el periodo anterior a la adopción del régimen de libre flotación se presentan en el cuadro $3 .{ }^{7}$ Cabe destacar que para la totalidad de los sistemas existe evidencia de cointegración y que el sentido de la relación entre las variables es positivo: de-

${ }^{7}$ En todos los casos los coeficientes resultaron significativos en niveles convencionales. No reportamos los estadísticos de prueba por brevedad. 
preciaciones (apreciaciones) del peso se asocian con alzas (disminuciones) en los precios. ${ }^{8}$ Las elasticidades en todos los casos son menores a la unidad, lo cual es consistente con lo que se encuentra en la literatura. Esto, fundamentalmente, indica que el traspaso no es completo, una depreciación de un punto porcentual conlleva a un incremento en la inflación de menos de un punto porcentual. Análisis como los realizados por Garcés (1999) y Conesa (1998) derivan resultados en este sentido, Garcés identifica una magnitud de traspaso de precios externos al precio de bienes comerciables de 0.81 ; la misma es menor para los bienes no comerciables. Conesa, por su parte, estima las elasticidades de traspaso para 47 sectores de la industria manufacturera. En todos los casos la magnitud es menor a la unidad.

\section{Cuadro 3}

\section{Pruebas de cointegración para el periodo 1992-1994}

\begin{tabular}{lcccc}
\hline \multicolumn{1}{c}{ Serie } & $\begin{array}{c}\text { Hipótesis } \\
\text { sobre }\end{array}$ & $\begin{array}{c}\text { Estadistico } \\
\text { de prueba }\end{array}$ & $\begin{array}{c}\text { Estadistico } \\
\text { de traza }\end{array}$ & $\begin{array}{c}\text { Vector de } \\
\text { cointegración }\end{array}$ \\
\hline IPC nacional & $\mathrm{r}=0$ & 38.29 & 25.87 & $1,-0.88$ \\
Frontera norte & $\mathrm{r}>1$ & 10.98 & 12.52 & \\
Noroeste & $\mathrm{r}=0$ & 36.53 & 25.87 & $1,-0.91$ \\
& $\mathrm{r}>1$ & 7.77 & 12.52 & \\
Noreste & $\mathrm{r}=0$ & 41.58 & 25.87 & $1,-0.89$ \\
& $\mathrm{r}>1$ & 10.65 & 12.52 & \\
Centro norte & $\mathrm{r}=0$ & 41.75 & 25.87 & $1,-0.89$ \\
& $\mathrm{r}>1$ & 10.58 & 12.52 & \\
Centro sur & $\mathrm{r}=0$ & 42.34 & 25.87 & $1,-0.90$ \\
& $\mathrm{r}>1$ & 11.06 & 12.52 & \\
Metropolitana & $\mathrm{r}=0$ & 40.56 & 25.87 & $1,-0.89$ \\
& $\mathrm{r}>1$ & 11.25 & 12.52 & \\
Sur & $\mathrm{r}=0$ & 38.68 & 25.87 & $1,-0.87$ \\
& $\mathrm{r}>1$ & 11.44 & 12.52 & \\
& $\mathrm{r}=0$ & 41.06 & 25.87 & $1,-0.89$ \\
\hline
\end{tabular}

Fuente: Elaboración propia.

En el cuadro 4 se muestran los resultados para el periodo posterior a la devaluación. Las elasticidades disminuyeron en comparación al periodo anterior. En promedio los coeficientes de cointegración son 0.14 puntos menores a lo que se encontró para el periodo de tipo de cambio fijo. Esto es consistente con lo que se identifica en Baqueiro et al. (2003) respecto a

\footnotetext{
${ }^{8}$ Se puntualiza que en los cuadros de resultados se reportan los coeficientes normalizados, por lo que la relación cualitativa entre las variables es la inversa al signo que aparece.
} 
que la magnitud del traspaso del tipo de cambio al nivel de precios tiende a decrecer a medida que la inflación desciende. ${ }^{9}$ Como se apreció en las gráficas anteriores, la tasa de crecimiento de los precios ha tendido a la baja desde que el sistema de tipo de cambio flexible fue adoptado.

\section{Cuadro 4}

\section{Pruebas de cointegración para el periodo 1996-2007}

\begin{tabular}{lcccc}
\hline \multicolumn{1}{c}{ Serie } & $\begin{array}{c}\text { Hipótesis } \\
\text { sobre }\end{array}$ & $\begin{array}{c}\text { Estadistico } \\
\text { de prueba }\end{array}$ & $\begin{array}{c}\text { Estadistico } \\
\text { de traza }\end{array}$ & $\begin{array}{c}\text { Vector de } \\
\text { cointegración }\end{array}$ \\
\hline IPC nacional & $\mathrm{r}=0$ & 38.91 & 25.87 & $1,-0.74$ \\
Frontera norte & $\mathrm{r}>1$ & 7.46 & 12.52 & \\
Noroeste & $\mathrm{r}=0$ & 47.57 & 25.87 & $1,-0.72$ \\
Noreste & $\mathrm{r}>1$ & 7.25 & 12.52 & \\
& $\mathrm{r}=0$ & 31.78 & 25.87 & $1,-0.77$ \\
Centro norte & $\mathrm{r}>1$ & 7.63 & 12.52 & \\
& $\mathrm{r}=0$ & 38.02 & 25.87 & $1,-0.78$ \\
Centro sur & $\mathrm{r}>1$ & 7.39 & 12.52 & \\
& $\mathrm{r}=0$ & 43.24 & 25.87 & $1,-0.76$ \\
Metropolitana & $\mathrm{r}>1$ & 7.08 & 12.52 & \\
& $\mathrm{r}=0$ & 40.91 & 25.87 & $1,-0.77$ \\
Sur & $\mathrm{r}>1$ & 7.24 & 12.52 & \\
& $\mathrm{r}=0$ & 53.41 & 25.87 & $1,-0.73$ \\
& $\mathrm{r}>1$ & 7.55 & 12.52 & \\
\hline
\end{tabular}

Fuente: Elaboración propia.

De igual forma, se debe notar que los coeficientes parecen estar sobreestimados, ya que en estudios previos la magnitud del traspaso del tipo de cambio a los precios es de alrededor de $0.60 .{ }^{10}$ En este caso se obtienen coeficientes para el primer periodo en el rango $[0.87,0.91]$ y de $[0.72,0.78]$ para el segundo. A pesar de ello, se plantea que los resultados son válidos y útiles para determinar si el efecto del tipo de cambio sobre el nivel de precios se modificó a partir de la adopción del régimen de libre flotación.

Más allá del cambio en el nivel de traspaso a través del tiempo, llama la atención que en el largo plazo dicho efecto parece no ser muy distinto a través de las diferentes regiones que se han analizado. En términos de

\footnotetext{
${ }^{9}$ De acuerdo con un estudio reciente de la CEPAL, la inflación en México sigue con una tendencia a disminuir como lo muestra el Estudio Económico de América Latina y el Caribe 2009-2010 (año).

${ }^{10}$ Véase Garcés (1999) o Esquivel y Razo (2003).
} 
números, la similitud entre las elasticidades tanto en el periodo de tipo de cambio fijo como en el de libre flotación se aprecia al notar que las desviaciones estándar son 0.01 y 0.02 , respectivamente. Esto indica que en horizontes amplios, una vez que los efectos transitorios se han disipado, el comportamiento de los precios tiende a homologarse. Este patrón pudiera no ser el mismo para el corto plazo, ya que la respuesta de los precios a choques transitorios del tipo de cambio pudiera tener efectos distintos en diversas regiones, tal y como se planteó en la sección introductoria. A continuación se evalúa esta posibilidad.

\subsection{Análisis de corto plazo}

Como se indicó en su oportunidad, para llevar a cabo las estimaciones de corto plazo se opta por instrumentar la prueba de ciclos comunes propuesta en Vahid y Engle (2003). Como lo han mostrado trabajos como Issler y Vahid (2001) y Herrera (2004), esta metodología, para estimar las relaciones de corto plazo entre series de tiempo, produce ganancias de eficiencia y en este sentido es superior a metodologías alternativas. En este caso, también se construyen sistemas bivariados con cada uno de los índices de precios y el tipo de cambio nominal; los resultados para los dos periodos de análisis se presentan en el cuadro 5. La hipótesis de la existencia de un ciclo común no se rechaza en ninguno de los sistemas para el periodo anterior a 1995. La relación cualitativa es la esperada: una depreciación del peso conlleva a un alza inmediata del nivel de precios; las magnitudes de las elasticidades de corto plazo son muy similares a las que se encontraron para el caso de cointegración. En un principio, quizá se hubiera esperado que los coeficientes fueran mayores, ya que a medida que pasa el tiempo la sensibilidad de los precios a cambios inesperados de los precios externos tiende a disminuir. Así, la respuesta instantánea pudiera ser mayor que la respuesta de largo plazo. Se debe reconocer, sin embargo, que el periodo de análisis coincide con episodios de fuertes depreciaciones de la moneda y significantes incrementos contemporáneos en los precios. De tal forma que resulta razonable obtener magnitudes como las que se muestran.

Para el periodo posterior a la crisis se obtienen resultados muy interesantes: primero, sólo en cuatro de las siete regiones analizadas se sigue observando una respuesta contemporánea del nivel de precios al tipo de cambio, esto es, su dinámica parece estar sincronizada, más aún para los casos en los que se encuentra una relación estadística significativa, los coeficientes son marcadamente menores a los que se obtuvieron para el periodo anterior a la modificación de régimen cambiario. Para el caso del índice nacional el coeficiente disminuye de 0.89 a 0.25 , un poco más 
de la tercera parte. De hecho, disminuciones similares se observan para las otras regiones. Una vez más, los resultados son consistentes con lo que se pudiera esperar, debido a que en el periodo posterior a 1995 la inflación ha experimentado una baja sostenida. Nótese finalmente que los vectores de comovimiento son similares entre ellos. El rango de magnitudes de coeficientes se encuentra entre 0.24 y 0.32 , esto sugiere que la respuesta del nivel de precios a movimientos transitorios del tipo de cambio a nivel nacional es parecida al de las regiones para las cuales se identifica ciclo común.

Llama la atención de manera particular el hecho de que ahora, para la frontera norte, la zona noroste y la zona sur, el nivel de precios y el tipo de cambio no comparten un ciclo común. En otras palabras no presentan movimientos transitorios similares. Esto se podría interpretar como evidencia de que los precios en estas zonas ya no responden significativamente a la dinámica de las fluctuaciones del tipo de cambio, al menos en el corto plazo; cuando el peso se deprecia, por ejemplo, los precios no suben instantáneamente, sino que toma un tiempo para que respondan al choque en el tipo de cambio. Por otro lado, el efecto sí es significativo en las otras zonas así como a nivel nacional, presentándose la mayor elasticidad en la región centro norte, 0.32 .

\section{Cuadro 5}

\section{Pruebas de ciclo común}

\begin{tabular}{|c|c|c|c|c|c|}
\hline \multirow[b]{2}{*}{ Serie } & \multirow[b]{2}{*}{$\begin{array}{l}\text { Hipótesis } \\
\text { sobres }\end{array}$} & \multicolumn{2}{|c|}{ 1982-1994 } & \multicolumn{2}{|c|}{$1996-2007$} \\
\hline & & Valor $p$ & $\begin{array}{c}\text { Vector de } \\
\text { comovimiento }\end{array}$ & Valor $p$ & $\begin{array}{c}\text { Vector de } \\
\text { comovimiento }\end{array}$ \\
\hline \multirow[t]{2}{*}{ IPC nacional } & $s>0$ & 0.22 & $1,-0.89$ & 0.12 & $1,-0.25$ \\
\hline & $s>1$ & 0.00 & & 0.00 & \\
\hline \multirow[t]{2}{*}{ Frontera norte } & $s>0$ & 0.09 & $1,-0.84$ & 0.02 & N/A \\
\hline & $s>1$ & 0.00 & & 0.00 & \\
\hline \multirow[t]{2}{*}{ Noroeste } & $s>0$ & 0.24 & $1,-0.89$ & 0.03 & N/A \\
\hline & $s>1$ & 0.00 & & 0.00 & \\
\hline \multirow[t]{2}{*}{ Noreste } & $s>0$ & 0.24 & & 0.11 & $1,-0.24$ \\
\hline & $s>1$ & 0.00 & $1,-0.88$ & 0.00 & \\
\hline \multirow[t]{2}{*}{ Centro norte } & $s>0$ & 0.30 & $1,-0.89$ & 0.10 & $1,-0.32$ \\
\hline & $s>1$ & 0.00 & & 0.00 & \\
\hline \multirow[t]{2}{*}{ Centro sur } & $s>0$ & 0.16 & $1,-0.88$ & 0.08 & $1,-0.30$ \\
\hline & $s>1$ & 0.00 & & 0.00 & \\
\hline \multirow[t]{2}{*}{ Metropolitana } & $s>0$ & 0.24 & $1,-0.90$ & 0.13 & $1,-0.30$ \\
\hline & $s>1$ & 0.00 & & 0.00 & \\
\hline \multirow[t]{2}{*}{ Sur } & $s>0$ & 0.20 & $1,-0.88$ & 0.03 & N/A \\
\hline & $s>1$ & 0.00 & & 0.00 & \\
\hline
\end{tabular}

Fuente: Elaboración propia. 
Identificar puntualmente los factores por los cuales los precios responden de manera diferente a variaciones en el tipo de cambio en el corto plazo no es trivial y, ciertamente, escapa el alcance del presente documento, a pesar de ello es posible proponer algunas posibilidades. Considere el típico canal de traspaso de tipo de cambio a los precios, que relaciona las variaciones en los términos de intercambio con los costos de insumos importados, de acuerdo con él, una depreciación conllevaría a un alza en dichos costos. Las empresas entonces buscarían alguna estrategia para traspasar ese incremento a los consumidores. En el largo plazo, el mecanismo parece operar tal y como se describe, ya que en los dos periodos considerados en el ejercicio de cointegración, la elasticidad de los precios al tipo de cambio resulta significativa y las series comparten una tendencia común. Para el corto plazo, sin embargo, en la zona fronteriza, noroeste y sur del país, el resultado no se aplica. Esto sugiere dos posibilidades: que la depreciación no afecta significativamente a los costos de producción, o que las empresas no tendrían el poder de traspasar los incrementos en sus costos a los consumidores.

Para el caso de la frontera se podría pensar en la primera posibilidad: choques transitorios en el tipo de cambio no afectan a los costos de producción instantáneamente, como es sabido, en esta región las empresas tienen una arraigada tradición de intercambio con Estados Unidos, por lo cual muy probablemente mantienen contratos de mediano o largo plazo con sus proveedores para protegerse de cambios inesperados en los términos de intercambio. Si este fuera el caso, entonces encontraríamos que en el corto plazo los precios no responden a los choques del tipo de cambio, pero sí lo hacen en horizontes más amplios, una vez que se ajustan los contratos. Así, la rigidez en los contratos que las empresas establecen con sus proveedores podría explicar por qué los precios no comparten movimientos transitorios con el tipo de cambio. Un argumento similar podría entenderse para la zona noroeste, donde se incluye a Hermosillo, que comercia intensivamente con la ciudad fronteriza de Nogales.

Con respecto a la zona sur la explicación de rigidez de contratos podría no aplicarse. En este caso se podría pensar en otra posibilidad: dadas las características de las industrias que se ubican en esta zona, posiblemente la cantidad de insumos importados que emplean no sea muy importante. Esto es, en los estados de Oaxaca y Chiapas, por ejemplo, el sector primario sigue siendo el motor de sus economías y por su naturaleza no emplea cantidades significativas de insumos importados. Por esto, cuando se presenta una depreciación, los costos de producción no tienen por que incrementarse contemporáneamente, y por ende los precios consumidor no tendrían que hacerlo. Así, el alza en los costos de los insumos 
en respuesta a una depreciación puede no ser instantánea, y sólo toma lugar en el largo plazo.

Cabe resaltar que los escenarios que se plantean representan, por supuesto, únicamente algunas posibilidades razonablemente factibles, es evidente que será necesario llevar a cabo un análisis más profundo al respecto; en el cual se tendría que considerar, por ejemplo, el segundo canal que mencionamos anteriormente, y que se refiere al poder que tienen las empresas para traspasar los incrementos en sus costos a los consumidores. Para el caso de la frontera norte, las condiciones de demanda son ciertamente distintas a otras regiones del país, ya que las empresas que se ubican en esta región no sólo compiten con empresas nacionales, sino con sus contrapartes estadounidenses. De tal forma que el poder de traspaso de los precios-productor a precios-consumidor es probablemente muy distinto en dicha región, en relación con el que tienen empresas ubicadas en otras partes del país.

\section{Conclusiones}

La inflación es un fenómeno que preocupa por sus efectos nocivos sobre la actividad económica. En el caso de México, estudios como Garcés (1999) y Esquivel y Razo (2003) han identificado que los salarios y el tipo de cambio constituyen sus determinantes más importantes. En este documento se analiza el segundo factor, el tipo de cambio, y se evalúa si su efecto sobre el nivel de precios ha cambiado a partir de la adopción del sistema de libre flotación. Consistente con resultados de estudios previos, como Baqueiro et al. (2003), se encuentra que el traspaso del tipo de cambio ha disminuido. Para el periodo anterior a la modificación del régimen cambiario la magnitud del traspaso era cercana a la unidad, mientras que después la misma se ubica alrededor de un tercio. Esto es cierto no sólo a nivel nacional, sino en cada una de siete regiones en las cuales se dividió el país: frontera norte, noroeste, noreste, centro norte, centro sur, y sur. La disminución se presenta tanto en el largo como en el corto plazo, en particular, los coeficientes de cointegración y ciclos comunes son menores para el periodo más reciente, esto es, con episodios de inflación controlada y estable. Destaca en el análisis que los choques transitorios del tipo de cambio no influyen significativamente en cada una de las regiones de análisis después de 1995. Para la zona fronteriza, del noroeste y del sur, se encuentra que el nivel de precios no responde significativamente a las variaciones transitorias del tipo de cambio, es decir, los índices de precios no comparten un ciclo común con la variable de tipo de cambio; cabe también destacar que en los casos para los cuales sí se identificó ciclo común, nivel nacional y cuatro regiones, las magni- 
tudes de la respuesta de los precios a variaciones del tipo de cambio son similares. Esto podría sugerir que la estructura socioeconómica de esas regiones es parecida a la que exhibe la economía agregada, así, le sería posible a aquéllos con la posibilidad de tomar decisiones que repercuten en el desarrollo social y económico de la población, anticipar la respuesta de los precios de cada región a fluctuaciones del tipo de cambio con base en lo que se observa a nivel nacional.

Se plantea que este resultado se debe a características socioeconómicas específicas de cada región, aparentemente, en algunas zonas del país, los precios son más rígidos y, por ende, responden más lentamente a efectos transitorios, esta rigidez se puede deber a los patrones de consumo y oportunidades de sustitución que pudieran existir, o a la flexibilidad de los procesos productivos ante un alza en el costo de los insumos importados; así, a partir de los resultados aquí obtenidos, se deja abierta la posibilidad de realizar estudios más especializados que se dediquen a identificar por qué el traspaso del tipo de cambio al nivel de precios es diferente en la república mexicana. Se podría investigar, por ejemplo, la estructura industrial en las diferentes regiones del país, esto ayudaría a entender cómo el tipo de cambio influye sobre los costos de producción y la capacidad de las empresas de traspasar cambios en sus costos a los precios que paga el consumidor. Naturalmente, dichos esfuerzos deberán considerar el periodo de libre flotación, ya que es en éste donde se encuentra que algunos niveles de precios no comparten ciclos comunes con el tipo de cambio.

\section{Bibliografía}

Baer, Werner (1967), "The inflation controversy in Latin America: A survey", Latin American Research Review, 2, Latin American Studies Association, San Francisco, pp. 3-25.

Baqueiro, Armando, Alejandro Díaz de León y Alberto Torres (2003), "¿Temor a la flotación o a la inflación? La importancia del traspaso del tipo de cambio a los precios", documento de investigación, Banco de México, México.

Banco de México (2008), "Índice nacional de precios al consumidor", Banco de México, www.banxico.org.mx, 1 de junio de 2008.

Castillo-Ponce, Ramón y Jorge Herrera (2008), "Short-run dynamics of the price level and external exposure: evidence from Mexico", 
International Journal of Applied Economics, 5 (1), Institute of International Economic Studies, Hammond, pp. 30-47.

Cepal (Comisión Económica para América Latina y el Caribe) (2010), "Estudio Económico de América Latina y el Caribe 2009-2010", CEPAL.

Conesa, Andrés (1998) "Pass-through del tipo de cambio y de los salarios: Teoría y evidencia para la industria manufacturera”, documento de investigación, Banco de México, México.

Dornbusch, Rudiger (1976), "Expectations and exchange rate dynamics", Journal of Political Economy, 84, The University of Chicago Press, Chicago, pp. 1161-1176.

Esquivel, Gerardo y Raúl Razo (2003), "Fuentes de la inflación en México, 1989-2000: Un análisis multicausal de corrección de errores", Estudios Económicos, 18, El Colegio de México, México, pp. 181-226.

Fullerton, Thomas y Cuauhtémoc Calderón (1999), "Inflationary pressure determinants in Mexico", Estudios Económicos, 14, El Colegio de México, México, pp. 33-51.

Garcés, Daniel (1999), "Determinación del nivel de precios y la dinámica inflacionaria en México", documento de investigación, Banco de México, México.

Harberger, Arnold (1963), "The dynamics of inflation in Chile", en Carl Christ y Yehuda Grunfeld (eds.), Measurement in Economics, Stanford University Press, California, pp. 219-252.

Herrera, Jorge (2004), "Business cycles in Mexico and the United States: do they share common movements?", Journal of Applied Economics, 7, Universidad del cema, Buenos Aires, pp. 303-323.

Holmes, Mark (2008), "Real exchange rate stationarity in Latin America and relative purchasing power parity: A regime switching approach", Open Economies Review, 19, New York, pp. 261-275.

Issler, Joao Victor y Farshid Vahid (2001), "Common cycles and the importance of transitory shocks to macroeconomic aggregates", 
Journal of Monetary Economics, 47, Elsevier, Maryland Hights, pp. 449-475.

Johansen, Soren (1991), "Estimation and hypothesis testing of cointegration vectors in gaussian vector autoregressive models", Econometrica, 59, The Econometric Society, New York, pp. 1551-1580.

Kamin, Steven y Marc Klau (2003), "A multi-country comparison of the linkages between inflation and exchange rate competitiveness", International Journal of Finance and Economic, 8, John Wiley and Sons, Ltd., Malden, pp. 167-184.

Lucas, Robert y Leonard Rapping (1969), "Real Wages, Employment and Inflation”, Journal of Political Economy, 77, The University of Chicago Press, Chicago, pp. 721-754.

Patterson, Kerry (2000), An introduction to applied econometrics a time series approach, Palgrave, New York.

Pérez-López, Alejandro y Moisés Schwartz (1999), "Inflación y ciclos económicos", documento de investigación, Banco de México, México.

Solow, Robert (1969), Price expectations and the behavior of the price level, University Press, Manchester.

Sonora, Robert (2005), "City CPI convergence in Mexico", Review of Development Economics, 9 (3), John Wiley and Sons, pp. 359-367.

Stock, James y Mark Watson (1988), "Testing for common trends", Journal of the American Statistical Association, 83, American Statistical Association, Washington DC., pp. 1097-1107.

Vahid, Farshid y Robert Engle (1993), "Common trends and common cycles", Journal of Applied Econometrics, 8, John Wiley and Sons, Ltd., Malden, pp. 341-360.

Recibido: 21 de enero de 2009. Reenviado: 24 de enero de 2011. Aceptado: 8 de noviembre de 2011. 
Ramón A. Castillo Ponce. Es doctor en economía por la Universidad de California, Irvine, actualmente es profesor-investigador de la Facultad de Economía y Relaciones Internacionales en la Universidad Autónoma de Baja California y profesor-investigador en el Departamento de Economía y Estadística en la Universidad Estatal de California, Los Ángeles. Es miembro del Sistema Nacional de Investigadores, nivel II. Su línea de investigación actual es aplicaciones de econometría de series de tiempo. Entre sus últimas publicaciones destacan, en coautoría, "Macroeconomic determinants of remittances for a dollarized economy: the case of El Salvador" Journal of Economic Studies, vol. 38 núm. 5, Emerald Group Publishing Limited, Bradford, pp. 562-576 (2011); en coautoría, "Synchronization of economic activity between Mexico and the United States: What are the causes?", Revista de Análisis Económico, 25 (1), Santiago de Chile, pp. 15-48 (2011); en coautoría, Econometría práctica: Fundamentos de series de tiempo, Universidad Autónoma de Baja California, México (2010).

Rogelio Varela Llamas. Es doctor en economía industrial y relaciones laborales por la Universidad de Castilla-La Mancha, España. Actualmente es profesor investigador de la Facultad de Economía y Relaciones Internacionales en la Universidad Autónoma de Baja California. Es miembro del Sistema Nacional de Investigadores, nivel I. Su línea de investigación actual es economía mexicana con énfasis en tópicos del mercado de trabajo. Entre sus últimas publicaciones destacan: en coautoría, "Determinantes de los ingresos salariales en México: Una perspectiva de capital humano" en Región y Sociedad, vol. xxir, núm. 49, El Colegio de Sonora, Sonora, pp. 117- 142 (2010); en coautoría, "Pronóstico del comportamiento del empleo en Baja California" en José Guadalupe Osuna Millán (coord.), Baja California ante la crisis, Gobierno del Estado de Baja California-Universidad Autónoma de Baja California, Baja California, pp. 119-149 (2011); en coautoría, Econometría práctica: Fundamentos de series de tiempo, Universidad Autónoma de Baja California, México (2010). "Determinantes de la divergencia regional en México", en Julia Hernández, y Javier Martínez (eds.), La economía mexicana regional en los albores del siglo XXI, Universidad Autónoma de Chihuahua, Chihuahua, pp. 169-202 (2010); en coautoría, "El trigo en Baja California”, Editorial Porrúa-Universidad Autónoma de Baja California, México (2010).

Juan Manuel Ocegueda Hernández. Es doctor en economía por la Universidad Nacional Autónoma de México. Actualmente es profesorinvestigador en la Facultad de Economía y Relaciones Internacionales en la Universidad Autónoma de Baja California y Jefe de la Unidad de 
Presupuesto y Finanzas en la misma institución. Es miembro del Sistema Nacional de Investigadores, nivel II. Su línea de investigación actual es crecimiento económico regional en México. Entre sus últimas publicaciones destacan: "Causas del lento crecimiento de la economía nayarita, 1993-2007” en Karla S. Barrón, Jesús A. Madera y Lourdes Pacheco (eds.), Nayarit. Economía y sociedad, Universidad Autónoma de Nayarit, Nayarit, pp. 39-62 (2012); en coautoría, "Estructura económica y tasa de crecimiento en la frontera norte de México", Problemas del desarrollo, vol. 42, núm. 164, México, pp. 71-97 (2011); en coautoría, "Pronóstico del comportamiento del empleo en Baja California" en José Guadalupe Osuna Millán (coord.), Baja California ante la crisis, Gobierno del Estado de Baja California-Universidad Autónoma de Baja California, Baja California, pp. 119-149 (2011); en coautoría, "Determinantes de los ingresos salariales en México: una perspectiva de capital humano" en Región y Sociedad, vol. xxir, núm. 49, El Colegio de Sonora, Sonora, pp. 117-142 (2010); "Trade liberalization, specialization and regional divergence in México", en Ana Bárbara Mungaray y David Ledezma (eds.), México and the world: comparative perspectives for the study development, University of California Los Ángeles-Universidad Autónoma de Baja California, México, pp. 124-155 (2010); en coautoría, "Determinantes de la divergencia regional en México”, en Julia Hernández, y Javier Martínez (eds.), La economía mexicana regional en los albores del siglo XXI, Universidad Autónoma de Chihuahua, Chihuahua, pp. 169-202 (2010). 